\title{
The effect of social Nervous (SaSo) exercise with insulin resistance (HOMA-IR) on gestational diabetes mellitus among Indonesian pregnant women: a quasi-experimental study
}

Effatul Afifah ( $\square$ effatulafifah@almaata.ac.id )

University of Alma Ata

Detty Siti Nurdiati

Universitas Gadjah Mada

Hamam Hadi

University of Alma Ata

Zainal Muttaqien Sofro

Universitas Gadjah Mada

Ahmad Hamim Sadewa

Universitas Gadjah Mada

Arif Sabta Aji

University of Alma Ata

\section{Research Article}

Keywords: Gestational diabetes mellitus, intervention, social nervous exercise, HOMA-IR, Indonesia

Posted Date: November 24th, 2021

DOI: https://doi.org/10.21203/rs.3.rs-1058455/v1

License: (c) (i) This work is licensed under a Creative Commons Attribution 4.0 International License.

Read Full License 


\section{Abstract \\ Background}

Gestational diabetes mellitus (GDM) is a carbohydrate intolerance condition that is diagnosed for the first-time during pregnancy and is associated with various adverse pregnancy outcomes for both mother and child, such as increased rates of perinatal complications and long-term morbidity. Exercise is a strategy to reduce hyperglycemia experienced during gestational diabetes mellitus. One type of exercise that can be done is a social nervous exercise (SaSo). SaSo can stimulate the parasympathetic or myelinated vagus nerves and control blood glucose by stimulating autonomic nerves system so that nerve homeostasis and glucose homeostasis occur. This study aimed to determine the impact of a SaSo program consisting of warm-up, core (prayer movements) and cool-down exercises on glucose homeostasis (HOMA-IR) parameters in women with GDM.

\section{Methods}

The study used a quasi-experimental design. Thirty-seven women with GDM at 24-28 weeks gestation were divided into an experimental group $(n=19)$ with a regularly supervised SaSo program $(n=18)$. The control group received only standard antenatal care for GDM. The exercise program started from the time of diagnosis of diabetes to six weeks of intervention conducted twice per week with sessions lasting 4045 minutes. Bivariate analysis was used to test the difference in means with pretest and posttest results.

\section{Results}

Majority of pregnant women were in the not at-risk age category for control and intervention groups (79\% and $83 \%$, respectively). The baseline data results for the experimental and control groups were homogeneous, with no difference in baseline variables $(P>0.05)$. The social nervous exercise experimental group had lower mean difference $(M D \pm S D)$ levels of insulin resistence in late pregnancy compared to the control group $(-9.15 \pm 10.06$ vs $3.42 \pm 14.84)(P=0.004)$.

\section{Conclusions}

A social nervous exercise program has a beneficial effect on insulin resistance (HOMA-IR) levels in late pregnancy. Further research needs to be done with larger studies to confirm the findings of this study.

\section{Trial registration:}

Ethical approval was obtained from the Medical and Health Research Ethics Committee of the Faculty of Medicine, Universitas Gadjah Mada Yogyakarta (KE/0978/08/2019). 


\section{Background}

Gestational diabetes mellitus (GDM) is a carbohydrate tolerance disorder that is usually first recognized during pregnancy at 24-28 weeks of gestation. GDM occurs due to physiological, endocrine, and metabolic changes during pregnancy in order to meet the nutrients and oxygen requirements of the fetus continuously. In GDM, the diabetogenic condition similar to that occurring in type 2 diabetes mellitus (T2DM) is created, increasing the insulin resistance, decreasing the insulin sensitivity and consequently enhancing the need for insulin [1]. It is believed that this will increase the prevalence of T2DM because risk factors continue to develop, especially obesity and lifestyle [2, 3].

The occurrence of GDM has been increasing in Asian countries recently. The prevalence of GDM in Korea in 2009 was $7.5 \%$ and continued to increase in 2011 to 9.5\% [3]. The prevalence of GDM in Asia is 11.5\% [4]. The prevalence of GDM in Indonesia is $1.9 \%-3.6 \%$ in general pregnancy [5]. Although this data is lower than the prevalence in the United States and India, the problem of GDM in Indonesia must be treated seriously. In the research from Soewondo and Pramono (2011), the GDM prevalence data was $12.9 \%$ in screening for GDM in pregnant women in 10 health centers in Bantul district, Yogyakarta, Indonesia [5].

GDM is associated with risk factors and insulin resistance. One of the factors that can control the increase in blood glucose during pregnancy is managing physical activity. When a person does physical activity, there will be muscle contractions which in turn will make it easier for blood glucose to enter the cells and reduce insulin resistance which results in lowering blood glucose levels [2]. Observational studies have shown that exercise can be a non-invasive therapeutic option to prevent and manage GDM that can be applied to the antenatal population. Previous studies have described the potential mechanism of exercise from the perspective of reducing insulin resistance, which is the root cause of $\operatorname{GDM}[6,7,2]$.

Guemes and Georgiou (2018) reviewed that the central nervous system (CNS) plays an important role in glucose homoeostasis through the control of pancreatic secretion and insulin sensitivity which could potentially be used as a pathway to improve glucose control [8]. Individuals with diabetes mellitus or GDM, hypertension and dyslipidemia have increased sympathetic nerve activity $[9,10,11]$. In both animals and humans, it has been shown that sympathetic nervous overactivity plays an important role in the etiology and complications of several metabolic conditions that make up the Metabolic Syndrome (MetS) group. Evidence also shows that excessive sympathetic activity causes two major metabolic changes of MetS, namely central obesity and insulin resistance [12].

The sympathetic nervous system (SNS) plays a role in cardiovascular and metabolic control. Sympathetic activation induces glycogenolysis, glucose production from the liver and increased lipolysis in adipose tissue, channeling lipid substrates towards gluconeogenesis, resulting in an increase in blood glucose. In the pancreas, SNS activation is associated with decreased insulin and increased glucagon levels in the portal circulation and in peripheral tissues such as skeletal muscles and adipose tissue. Sympathetic nerve-mediated vasoconstriction in peripheral arterioles leads to impaired glucose uptake and insulin resistance [10]. 
The increase in sympathetic nerves in individuals needs to be inhibited by increasing parasympathetic nerve activity so that there will be a balance in the autonomic nerves [13]. According to the Polyvagal Theory, the myelinated parasympathetic nerves $(\mathrm{V}, \mathrm{VII}, \mathrm{IX}, \mathrm{X}$ and $\mathrm{XI})$ play a role in social communication, which function to calm oneself and inhibit the influence of the adrenal-sympathetic and hypothalamicpituitary-adrenal (HPA) axis. Parasympathetic nerve stimulation will reduce sympathetic nerve activation [14]. Parasympathetic nerve stimulation can stimulate the pancreas and insulin through stimulation of acetylcholine receptors [15]. Based on the findings of Porges, the researchers developed a set of exercises that can stimulate the parasympathetic nerves and can inhibit sympathetic nerve activity. As a result, these barriers can increase glucose homeostasis, so that it is expected to decrease blood glucose $[13,14$, 16].

The purpose of this study was to analyze the effectiveness of the social nervous exercise intervention (SaSo) on model assessment-estimated insulin resistance (HOMA-IR) and analyze data on sociodemographic characteristics, food intake, physical activity, pregnancy body mass index (BMI), health conditions of pregnant women and pregnancy history in women with GDM in Yogyakarta, Indonesia.

\section{Methods}

\section{Study design}

This study using a quasi-experimental design with pretest-posttest control group which was conducted in October 2019-April 2020. Two primary health care centers in Bantul, namely Banguntapan 2 and Sewon 2 were chosen to be the locations of this study based on the prevalence of women with GDM in the 2019 study. This research was conducted in two stages; the first stage of research was done with the survey method by conducting a screening test to detect women with GDM. This screening was done by checking fasting blood glucose in pregnant women after fasting for 8-10 hours, then followed by an OGTT blood glucose test. Based on the calculation of the sample, 20 women with GDM were needed, plus $10 \%$ of the anticipated loss of pregnant women in each group.

The second stage of the study was to conduct an experimental research design, namely a quasiexperimental with pretest-posttest control group aimed at evaluating the intervention by analyzing the causes and effects of the intervention on the research outcomes, but not using randomization.

Randomization was not possible due to health ethics reasons, where all the women with GDM had the right to participate in social nervous exercise interventions because they experienced an increase in blood glucose. At the beginning of the study, the researcher explained the aims, objectives, positive and negative impacts of the intervention and determined those with willingness to participate in the intervention. The SaSo intervention program also had its own subject criteria according to the guidelines and all samples had the same opportunity to be included in the intervention group and control group.

\section{Subject characteristics}


The subjects of this study were 19 respondents in the intervention group and 18 respondents in the control group. The research instruments used were as follows: The basic characteristic questionnaire of the respondents included: age, education level, type of occupation, family history of DM, parity, impaired

glucose tolerance, history of babies born with birth weight $>4,000 \mathrm{~g}$. The respondent's anthropometric and clinical data questionnaire included: current weight, height status, body mass index (BMI), and blood pressure. The Semi Quantitative Food Frequency Questionnaire (SQ-FFQ), and a food photo book Standard Riskesdas (2013) were used to estimate the food intake consumed by the pregnant women with an in-depth interview guide. ACCU Check (Performa) (Roche Diabetes Care GmbH 68305 Mannheim Germany) was used for checking fasting blood sugar levels at the time of screening for GDM. Blood collection used venous blood, while the enzymatic glucose measurement used the GOD-PAP method derived from venous blood measured before and after the exercise intervention. The International Physical Activity Questionnaire (IPAQ) questionnaire was used regarding the type and duration of physical activity. Measuring height (Microtoise) and weight used a stepping scale (OneMed), and a sports video was shown as a guide for exercise implementation for the mothers with GDM.

\section{Data collection}

Purposive sampling was done in collecting the research data. Inclusion criteria included pregnant women who had a pregnancy check-up at 24-28 weeks of gestation by examining fasting plasma glucose and oral glucose tolerance test (OGTT), pregnant women diagnosed with GDM with a fasting plasma glucose value of $92 \mathrm{mg} / \mathrm{dL}$ followed by OGTT $\geq 140 \mathrm{mg} / \mathrm{dL}$, pregnant women who had never had diabetes before pregnancy and were diagnosed diabetes mellitus for the first time in pregnancy, willing to be a research respondent stated by filling out an informed consent form, and Muslim faith. Exclusion criteria included: contraindications to exercise for more than 30-45 minutes performed more than 2 times/week, pregnant women following other exercise programs, pregnant women with heart disease, or chronic kidney disease, medications that affect glucose tolerance (drug administration), which affect blood glucose levels such as: glibenclamide, metformin and other drugs in the treatment of diabetes), and multiple pregnancies.

\section{HOMA-IR measurement}

HOMA-IR score reflects whole-body insulin resistance which was calculated by using the mathematical equation from Matthew from plasma insulin insulin in $\mathrm{mU} / \mathrm{ml}$ (milli-international units $/ \mathrm{ml}$ ) * glucose in $\mathrm{mmol} / \mathrm{L}$ concentrations divided by 22.5 [17].

\section{Intervention study}

The experimental group had the intervention with structured SaSo exercises twice per week along with standard prenatal care. The exercise program began one week after the diagnosis of GDM, and was continued for up to six weeks. Subject attendance was recorded at each training session. The minimum total duration of the SaSo exercise program was set at a minimum of six weeks. Minimum attendance was set at $80 \%$. Pregnant women in the control group only received standard care for GDM only. 
Each training session lasted 40-45 minutes. Movement steps in the SaSo consist of several movement sessions, including: 1). Warm-up movements, for 5-10 minutes, are done in a sitting and lying position. The warm-up session consists of stretching the pelvic muscles, back and a combination of yoga movements with breathing settings, accompanied by an ambient musical instrument, namely Kitaro music; 2). Core movements accompanied by instrumental music, core exercises (20-25 minutes) that include: The 1 st core movement is a complete prayer movement, which is a movement of posture, gestures and breathing. The 2 nd core movement is the repetition of the 1 st core movement which is done three times, and the 3rd core movement that includes: three therapies, namely 1) Listening Therapy, this therapy allows pregnant women to listen to the morotal Al-Quran Surah Ar-Rahman by doing the respiratory cycle (deep breath); 2) Vocalization therapy is applied by reading the Qur'an (short letters AlIkhlas, Al-Falaq and An-Nas) repeated three times, pregnant women read the Qur'an together with a loud voice; and 3) Smile Therapy, pregnant women make smiles by involving the heart with musical instruments which makes them happy and smiling accompanied by a song made by a goose or duck, and the last one is a cool-down movement, which is a relaxation period to end the session (10 minutes). This final movement is performed in a sitting and lying position. The SaSo is a combination of prayer movements with breathing exercises and yoga postures and combines all major muscle groups. During the exercise sessions, the pregnant women are provided with sufficient drinking water to prevent dehydration. The SaSo exercises are done using yoga mats and blocks as a seat for the sitting position.

\section{Data analysis}

All numeric data are presented in the mean with standard deviation (SD) and categorical data are presented in frequency ( $\mathrm{n}$ ) and percentage (\%). Univariate analysis was used to analyze the respondents' characteristic data such as age, nutritional status, hypertension status, history of hypertension, parity, family history of DM, history of macrosomia, education and occupation.

The data normality test was conducted to determine whether the data were normally distributed or not. The normality test of the data was done with the Kolmogorov Smirnov test for the group with the number of samples $(n>50)$ and the Shapiro Wilk test for the group with the number of samples $(n<50)$. If the $P$ value was significant ( $p \geq 0.05)$, then the data were considered normally distributed, and if $(p \leq 0.05)$, then the data were considered not normally distributed.

Bivariate analysis was used to test the difference in means with pretest and posttest results in one group using paired sample t-tests. Meanwhile, to test the mean difference with the pretest and posttest designs between the two groups, namely the intervention and control groups, if the data were normally distributed then independent sample t-tests were used and the Mann Whitney Test for the data not normally distributed. Data analysis used STATA version 13.1 special edition software.

\section{Results}

Screening results obtained 45 women with DMG. The pregnant women were offered whether they were willing to take part in the study and were willing to do exercise as the form of intervention given. A total of 
24 pregnant women $(n=24)$ who were willing to participate in the SaSo exercises were included in the intervention group, and there were 21 women with GDM ( $n=21)$ not willing to participate in the SaSo exercises who were included in the control group. However, there were eight pregnant women who dropped out during the data collection process so that the number of participants that could be analyzed completely included 19 in the intervention group and 18 in the control group (Figure 1).

\section{Subject Characteristics}

The characteristics of the pregnant women are presented in Table 1. The largest percentage of pregnant women's age status were in the not at-risk age category with 17 respondents $(89.5 \%)$ in the intervention group and 13 respondents $(72.2 \%)$ in the control group. The maternal nutritional status data indicated most were in the overweight category, namely 12 respondents (63.2\%) in the intervention group and 10 respondents $(55.6 \%)$ in the control group. The hypertension status in most of the pregnant women was in the non-hypertensive category, namely 17 respondents (89.5\%) in the intervention group, and 13 respondents $(72.2 \%)$ in the control group.

The largest history of hypertension data was in the non-hypertensive category, namely 16 respondents (84.2\%) in the intervention group, while 17 respondents (94.4\%) in the control group. The parity data indicated most mothers were in the primipara category, namely 11 respondents (57.8\%) in the intervention group, and in the control group, most were in the nullipara category, with 9 respondents (50.0\%).

\section{Changes in Average IR HOMA Levels Before and After Intervention}

The results of the HOMA-IR data analysis showed that in the intervention group the mean HOMA-IR level decreased significantly between before and after the intervention with $p<0.05$. Meanwhile, the control group showed an increase in HOMA IR levels $(p>0.05)$ between before and after the intervention, as shown in Table 2.

\section{The Effectiveness of Social Nervous Exercise on HOMA- IR Levels}

Table 3 shows the results of the analysis of the mean HOMA-IR in the intervention group were significantly different from the control group $(p=0.004)$. In the intervention group there was a decrease in the mean HOMA-IR, while in the control group the mean HOMA-IR increased.

\section{Discussion}


The results of base line data analysis showed that the majority of respondents in the intervention group $(89.5 \%)$ and the control group $(72.2 \%)$ were in the safe age range during pregnancy (20-35 years). If a woman becomes pregnant at the age of $<20$ years or $>35$ years, then the risk of experiencing complications in pregnancy will be greater. Previous research has shown that women who become pregnant at the age of 35 or less than 17 years have a greater risk of experiencing severe maternal morbidity compared to women who become pregnant at the age of 25-29 years [18]. In this study, the nutritional status of the majority of pregnant women belonged to the overweight category (BMI $>25.0$ $\mathrm{kg} / \mathrm{m} 2$ ), with $63.2 \%$ in the intervention group and $55.6 \%$ in the control group. The remaining $31.6 \%$ and $44.4 \%$ of respondents had normal nutritional status in the intervention and control groups, while there were only 1 respondent or $5.3 \%$

in the intervention group and $0 \%$ in the control group with underweight status. Nutritional status during pregnancy is one indicator that can affect pregnancy and pregnancy outcomes. Previous research has shown that underweight and overweight/obese conditions can have an adverse effect on pregnancy [19].

Concerning the hypertension status, $89.5 \%$ of respondents did not experience hypertension in the intervention group while for the control group, it was $72.2 \%$. Most respondents had no history of hypertension, with $84.2 \%$ in the intervention group and $94.4 \%$ in the control group. Some research results explained that the status of hypertension in pregnancy will adversely affect pregnancy. In the United States, chronic hypertension affects $1-5 \%$ of pregnancies, and it is known that GDM complicates $1-14 \%$ of pregnancies. Pregnancy can become complicated by hypertension and gestational hypertension is associated with increased maternal mortality and perinatal morbidity. Adverse outcomes associated with hypertension include: premature birth, caesarean section, fetal growth restriction, perinatal death, preeclampsia, acute renal failure, edema, and stroke or maternal death [20].

The results of parity analysis also showed that about $40-50 \%$ of respondents in the intervention and control groups were in primiparous parity status. Several studies have shown the effect of parity on increasing blood glucose. Fasting plasma glucose levels increase with increasing number of live births. Parity status has a significant relationship with GDM. Women who have had two, three, and four or more live births have 1.35 times higher risk, and the risk of diabetes is significantly higher than women who had one live birth [21]..

Respondents with a family history of DM were $10-30 \%$. Several studies have stated that there is a significant relationship between a family history of DM and GDM. The prevalence of GDM was significantly associated with a family history of $\operatorname{DM}(22,23,24]$. Family history of DM has a significant association with the risk of macrosomia and cesarean delivery during pregnancy [25]. Research data showed that there is a history of macrosomia in about $5.6-10.5 \%$ of respondents who have a history of giving birth to a macrosomia baby in a previous pregnancy. An overview of education and employment data indicated an average of $60-80 \%$ of respondents with a high school education or equivalent (SMA), and $20 \%$ who have a high education. As many as $70-80 \%$ of respondents work as housewives, and the rest are civil servants and entrepreneurs. Respondents with this level of education will have a sufficient 
level of awareness and knowledge to accept interventions and education provided in order to improve health status of pregnant women.

\section{Effects of social nervous exercise on insulin resistance (HOMA-IR)}

Patients with hypertension and dyslipidemia have increased sympathetic nerve activity (9). Flaa et al. [26] concluded that sympathetic activity precedes prediabetes and the development of insulin resistance. Increased sympathetic activity in healthy adults leads to insulin resistance, mainly through reduced blood flow. The increase in sympathetic nerves needs to be inhibited by increasing parasympathetic nerve activity so that there will be a balance in the autonomic nerves [13]. One way to increase the activity of the parasympathetic nervous system is to do SaSo, which are exercise movements that aim to stimulate the parasympathetic nerves by inhibiting sympathetic nerve activity in achieving glucose homeostasis. The SaSo involve low-intensity movements exercising the autonomic nerves that regulate balance in the body. The SaSo exercises can stimulate the myelinated parasympathetic nerve (vagus nerve/ $X$ ) which is supported by the V, VII, IX and XI cranial nerves, These are collectively referred to as the social nerves, namely the nerves: $\mathrm{V}, \mathrm{VII}, \mathrm{IX}, \mathrm{X}$ and $\mathrm{XI}$, which are connected through the corticobulbaris pathways [13].

Table 3 shows that social nervous exercise can reduce insulin resistance levels, possibly through two mechanisms: 1) stimulation of the parasympathetic nervous system as a social nerve consisting of nc $V$, VII, IX, X and XI which has an impact on autonomic nervous homeostasis resulting in glucose homeostasis and decreased insulin resistance (HOMA-IR); and 2) a mechanism through increased glucose uptake at the muscle and adipose cell levels. This description is supported by previous research which showed that autonomic nervous balance can reduce blood glucose levels [8]. The theory of autonomic nerves, is used to explain the mechanism of activation of the social nerve (vagus myelin) to create a relaxed and pleasant atmosphere during social nervous exercises. This theory is supported by the Polyvagal Theory introduced by Porges in 2001. The Polyvagal Theory is a theory that emphasizes the phylogenetic origin of the brain structures that regulate social nerves. This theory states that the evolution of the mammalian autonomic nervous system provides a neurophysiological layer for emotional experience and affective processes that are the main components of social behavior. This theory provides a biological explanation of the role of social, emotional and communication behavior [13].

Researchers suspect several theories are behind the possible mechanisms of social neural exercise in stimulating autonomic nervous homeostasis, among others: 1) social neural exercise can stimulate autonomic nervous homeostasis, through the mechanism of inhibiting the influence of sympathetic nerves to the heart and dampening the HPA axis so that by reducing sympathetic nerve activity and creating a state of calm behavior, while the sympathetic nerve control shifts to the parasympathetic nervous system so that it affects the balance of the autonomic nervous system [14]; 2) the mechanism of peripheral cholinergic regulation, where neurotransmitters release $A C h$, which will diffuse to its receptors, namely muscarinic receptors, on pancreatic cells muscarinic receptors (m3AChR) will bind to 
acetylcholine, triggering the secretion of pancreatic endocrine hormones resulting in increased production of insulin, glucagon and an increase in the number of beta cells, which causes a decrease in blood glucose levels (glucose homeostasis)[8]; 3) non-cholinergic mechanism, where neuropeptide-mediated neurotransmitters such as vasoactive intestinal polypeptide/Vasoactive intestinal polypeptide (VIP), pituitary adenylate cyclase activating polypeptide peptide/Pituitary adenylate cyclase activating polypeptide (PACAP), or gastrin-releasing peptide/gastrin-releasing peptides (GRP) thereby increasing pancreatic endocrine hormonal secretion [8]. 4) SaSo exercises can stimulate autonomic nervous homeostasis through the stimulation of the role of neuroception [16]; 5) SaSo exercises activate the fusiform face area (FFA) receptors by responding to facial expressions of pregnant women, where FFA activation is made by one of the neurons called "face cells" in the temporal section [27]; 6) SaSo exercises can create effective communication between pregnant women so as to create a comfortable and pleasant environment, which will stimulate the social nerves as a form of response from the social engagement system so that it affects blood glucose homeostasis [13], and 7) SaSo exercises stimulates pregnant women smiling, while the smiling and shared humor can create a relaxed and calm atmosphere so as to increase the effectiveness of stress management in GDM mothers [16].

The results of this study are supported by previous studies stating that parasympathetic nerve stimulation can stimulate the pancreas and insulin through stimulation of acetylcholine receptors $[15,8]$. These results are consistent with those observed by Shimazu et al. [28] who found electrical stimulation of the peripheral parasympathetic vagus nerves innervating the liver, which demonstrated parasympathetic control of the LHN (lateral hypothalamic nucleus) via hepatic glycogenesis. One study conducted by Güemes and Georgiou [8] concluded that electrical stimulation of the parasympathetic nerves can reduce postprandial blood glucose levels so that glucose homeostasis occurs. Experimental studies in have shown that transcutaneous auricular vagus nerve stimulation (taVNS) taVNS significantly reduced the two-hour glucose tolerance and systolic blood pressure over time compared with sham taVNS (29). Exercise can improve the balance of cardiac autonomic nerves, namely, increase parasympathetic and reduce cardiac sympathetic regulation and increase heart rate variability [30]. Research conducted by Alessio et al. concluded that activation of the parasympathetic nerves during the absorption phase of food contributes significantly to the postprandial insulin secretory response [31].

Safe conditions, individuals will experience glucose homeostasis and decreased insulin resistance, and this occurs because the social nerves can be activated through chanting the holy verses of the Koran and singing which is the vocalization of Sc IX and X, reciting Quranic verses and music (face to face expression or Sc VII and $\mathrm{V}$ ). The rotation and head movements when doing sports and prayer movements sc XI are a series of movements that are able to create pleasant conditions [32]. This is the role of neuroception. Neuroception in the temporal cortex can respond to sounds and faces. Neuroception regulates a person's behavior and physiological conditions to support adaptive behavior in dealing with safe and unsafe environmental conditions [13].

Physiologically, there are stages of communication in SaSo exercises. The communication involves the recitation of verses in the Qur'an, which is a form of individual communication with God. The 
communication is triggered by the activation of FFA which occurs due to a combination of verbal communication (spoken language) which is innervated by cranial nerve $\mathrm{V}$, while non-verbal forms of social nerve stimulation in the form of head, nape, and prostration movements are movements innervated by cranial nerves $\mathrm{XI}$, mimicking facial expressions and smiles in doing sports where the social nerves are innervated by cranial nerve VII, while the para-verbal communication (sounds that accompany words, such as intonation, tone of voice, tone, rhythm and verbal flow). The para-verbal form in social nerve activation is in the form of pronunciation and mahrojul letters in reading the Qur'an which will activate the IX cranial nerve. This stage of effective communication will stimulate the social nerves as a form of response from the social engagement system so that it affects blood glucose homeostasis [16]. This effective communication occurs between pregnant women and between facilitators and pregnant women.

Exercise can decrease circulating glucose and insulin during an exercise session and the period after. The suggested benefits of exercise during pregnancy include reduced low back pain, fluid retention and cardiovascular stress. The SaSo exercises are believed to play a role in reducing the risk of complications such as preterm birth and preeclampsia, and may help prevent pregnancy overweight and weight retention after delivery. Repetitive physical activity (exercise training) results in a sustained increase in insulin action on skeletal muscle in obese and insulin-resistant individuals. The molecular mechanism for increased glucose uptake with exercise training has been associated with increased expression and signaling activity of proteins as key involved in the regulation of glucose uptake and metabolism in skeletal muscles [6].

Evidence to support this study is shown in several observational studies that found pre-pregnancy exercise and exercise during pregnancy are associated with a decrease in insulin resistance. Which has an impact on a reduced risk of developing GDM [6]. Yoga practice also helps in reducing blood glucose because it improves blood circulation and increases insulin sensitivity resulting in increased utilization of glucose by muscle cells [33].Yoga diet and practice enhances the mind-body connection which results in physical and mental relaxation and reduction of physical and mental stress. Yoga practice has a positive effect on health through mechanisms of humoral activity and the nervous system [34]. Therefore, it is useful in lowering blood glucose [35].

\section{Conclusions}

A social nervous exercise program has a beneficial effect on insulin resistance (HOMA-IR) levels in late pregnancy. It is expected that pregnant women can pay more attention to risk factors for gestational diabetes mellitus, which include the lack of physical activity. Increased physical activity with the social nervous exercise intervention is expected to control blood glucose homeostasis of gestational diabetes mellitus.

\section{Abbreviations}


SaSo

social nervous exercise

GDM

gestational diabetes mellitus

DM

diabetes mellitus

T2DM

type 2 diabetes mellitus

HOMA-IR

model assessment-estimated insulin resistance

SNS

sympathy nervous system

BMI

body mass index

OGTT

oral glucose tolerance test

SQ-FFQ

semi quantitative food frequency questionnaire

IPAQ

international physical activity questionnaire.

\section{Declarations}

\section{Acknowledgement}

Authors want to thank the subjects who volunteered to participate in the study. We also thank for all research assistants, nutritionist in each primary health care, and cadre who contributed to the collection of data.

\section{Availability of data and materials}

The study datasets are available from the corresponding author on request.

\section{Author's contribution}

All authors (E.A, H.H, D.S.N, Z.M.S, and A.H.S) contributed to the design of the study, and E.F contributed to the collection of data. E.F and A.S.A were responsible for the statistical analysis, the interpretation of data, and wrote the first draft of manuscript, with help from D.S.N, A.H.S, Z.M.S and H.H who critically 
revised the text. All authors approved the final version of the manuscript and gave their consent for publication.

\section{Ethics approval and consent to participate}

Ethical approval was obtained from the Medical and Health Research Ethics Committee of the Faculty of Medicine, Universitas Gadjah Mada Yogyakarta (KE/0978/08/2019). The subjects in this study had completed an informed consent from before conducting the study. The research process upheld the Helsinki Declaration to meet the standards of research conducted with good ethical practices.

\section{Competing interest}

Authors have no conflict of interest in this study.

\section{Funding}

Funding was provided from the Indonesia Endowment Fund for Education (LPDP) of Republic of Indonesia Ministry of Finance.

\section{References}

1. Mottola MF, Artal R. Early Human Development Fetal and maternal metabolic responses to exercise during pregnancy. Early Human Development. 2016;1-9.

2. Amiri N, Metab D, Amiri FN, Sepidarkish M, Shirvani MA, Habibipour P. The effect of exercise on the prevention of gestational diabetes in obese and overweight pregnant women: a systematic review and meta - analysis. Diabetology \& Metabolic Syndrome. 2019;1-14.

3. Koo BK, Lee JH, Kim J, Jang EJ, Lee CH. Prevalence of gestational diabetes mellitus in Korea: A national Health Insurance database study. PLoS ONE. 2016;11(4):1-10.

4. Lee KW, Ching SM, Ramachandran V, Yee A, Hoo FK, Chia YC, et al. Prevalence and risk factors of gestational diabetes mellitus in Asia: a systematic review and meta-analysis. 2018;1-20.

5. Soewondo P, Pramono LA. Prevalence, characteristics, and predictors of pre-diabetes in Indonesia. 2011;20(4):283-94.

6. Wang $\mathrm{C}$, Janese $\mathrm{K}$, Yang $\mathrm{H}$. ScienceDirect Exercise and its role in gestational diabetes mellitus. Chronic Diseases and Translational Medicine. 2016;2(4):208-14.

7. Laredo-aguilera JA, Gallardo-bravo M, Rabanales-sotos JA. Physical Activity Programs during Pregnancy Are E ff ective for the Control of Gestational Diabetes Mellitus. 2020; 
8. And AG, Georgiou P. Review of the role of the nervous system in glucose homoeostasis and future perspectives towards the management of diabetes. 2018;1-18.

9. Couck MDE, Mravec B, Gidron Y. You may need the vagus nerve to understand pathophysiology and to treat diseases Clinical Science. 2012;(January 2014).

10. Carnagarin R, Matthews VB, Herat LY, Ho JK, Schlaich MP. Autonomic Regulation of Glucose Homeostasis: a Specific Role for Sympathetic Nervous System Activation. 2018;

11. Birmingham K, Gradinaru V, Anikeeva P, Grill WM, Pikov V, Mclaughlin B, et al. Bioelectronic medicines: a research roadmap. Nature Publishing Group. 2014;13(6):399-400.

12. Thorp AA, Schlaich MP. Relevance of Sympathetic Nervous System Activation in Obesity and Metabolic Syndrome. 2015;2015.

13. Porges SW. The polyvagal theory: phylogenetic substrates of a social nervous system. Zeitschrift fur Sozialpsychologie. 2001;32(1):1-11.

14. Porges SW. The polyvagal theory: New insights into adaptive reactions of the autonomic nervous system. 2011;76(Suppl 2).

15. Kaneto A, Miki E, Kosaka K. Effects of Vagal Stimulation on Glucagon and Insulin Secretion. 2015; (November).

16. Porges SW. Comprehensive Psychoneuroendocrinology Polyvagal Theory: A biobehavioral journey to sociality. Comprehensive Psychoneuroendocrinology. 2021;7(May):100069.

17. Matthews DR, Hosker JP, Rudenski AS, Naylor BA, Treacher DF, Turner RC. Homeostasis model assessment: insulin resistance and beta-cell function from fasting plasma glucose and insulin concentrations in man. Diabetologia. 1985 Jul;28(7):412-9.

18. Sheen J, Wright JD, Goffman D, Kern-goldberger AR, Booker W, Ms ZS, et al. Maternal age and risk for adverse outcomes. The American Journal of Obstetrics \& Gynecology. 2018;219(4):390.e1-390.e15.

19. Ramakrishnan U, Grant F, Goldenberg T, Zongrone A, Martorell R. Effect of Women 's Nutrition before and during Early Pregnancy on Maternal and Infant Outcomes: A Systematic Review. 2012;26:285301.

20. Braunthal S, Brateanu A. Hypertension in pregnancy: Pathophysiology and treatment. 2019;

21. Tian Y, Shen L, Wu J, Chen W, Yuan J, Yang H, et al. Parity and the Risk of Diabetes Mellitus among Chinese Women: A Cross-Sectional Evidence from the Tongji- Dongfeng Cohort Study. 2014;9(8):49.

22. Moosazadeh M, Asemi Z, Lankarani KB, Tabrizi R, Maharlouei N, Naghibzadeh-tahami A, et al. Diabetes \& Metabolic Syndrome: Clinical Research \& Reviews Family history of diabetes and the risk of gestational diabetes mellitus in Iran : A systematic review and meta-analysis. Diabetes \& Metabolic Syndrome: Clinical Research \& Reviews. 2016;(2):10-5.

23. Groof Z, Garashi G, Husain H, Owayed S, Albader S, Mouhsen H, et al. Prevalence, Risk Factors, and Fetomaternal Outcomes of Gestational Diabetes Mellitus in Kuwait : A Cross-Sectional Study. $2019 ; 2019$. 
24. Erem C, Kuzu UB, Deger O, Can G. Prevalence of gestational diabetes mellitus and associated risk factors in Turkish women: The Trabzon GDM Study. Archives of Medical Science. 2015;11(4):72435 .

25. Levy A, Wiznitzer A, Holcberg G, Mazor M, Sheiner E. Family history of diabetes mellitus as an independent risk factor for macrosomia and cesarean delivery. 2010;23(February):148-52.

26. Flaa A, Aksnes TA, Kjeldsen SE, Eide I, Rostrup M. Increased sympathetic reactivity may predict insulin resistance: an 18-year follow-up study. 2008;57:1422-7.

27. Kanwisher N, Mcdermott J, Chun MM. The Fusiform Face Area: A Module in Human Extrastriate Cortex Specialized for Face Perception. 1997;17(11):4302-11.

28. Nonogaki K, Iguchi A. Stress, Acute Hyperglycemia, and Hyperlipidemia 192 01997,. 1997;8(5):1927.

29. Huang F, Dong J, Kong J, Wang H, Meng H, Spaeth RB, et al. Effect of transcutaneous auricular vagus nerve stimulation on impaired glucose tolerance: a pilot randomized study. 2014;14(1):1-8.

30. Rossi J, Santama P, Airaksinen MS, Herzig K. Parasympathetic Innervation and Function of Endocrine Pancreas Requires the Glial Cell. 2005;54(May).

31. Alessio DAD, Kieffer TJ, Taborsky GJ, Havel PJ. Activation of the Parasympathetic Nervous System Is Necessary for Normal Meal-Induced Insulin Secretion in Rhesus Macaques *. 2001;86(3):1253-9.

32. Mccorry LK. TEACHERS ' TOPICS Physiology of the Autonomic Nervous System. 2007;71(4).

33. Balaji PA, Varne SR. Physiological effects of yoga asanas and pranayama on metabolic parameters, maternal, and fetal outcome in gestational diabetes. 2017;7(7):724-8.

34. Kuntsevich V, Bushell WC, Theise ND. Mechanisms of Yogic Practices in Health, Aging, and Disease. 2010;559-69.

35. Youngwanichsetha S, Phumdoung S, Ingkathawornwong T. The effects of mindfulness eating and yoga exercise on blood sugar levels of pregnant women with gestational diabetes mellitus. Applied Nursing Research. 2014;27(4):227-30.

\section{Tables}

Table 1. Characteristics of Pregnant Women in the Intervention and Control Groups. 


\begin{tabular}{|c|c|c|c|c|c|}
\hline \multirow[t]{3}{*}{ Characteristics of Pregnant Women } & & \multirow[t]{3}{*}{$P$ value } \\
\hline & & & \multicolumn{2}{|c|}{ Control $(\mathrm{N}=18)$} & \\
\hline & $\mathbf{n}$ & $\%$ & $\mathbf{n}$ & $\%$ & \\
\hline Age & & & & & 0.734 \\
\hline At Risk <20 \& >35 & 4 & 21.05 & 3 & 16,67 & \\
\hline No Risk 20-35 & 15 & 78,95 & 15 & 83,33 & \\
\hline Nutritional Status (BMI) & & & & & 0.486 \\
\hline Underweight & 1 & 5.3 & 0 & 0.0 & \\
\hline Normal & 6 & 31.6 & 8 & 44.4 & \\
\hline Overweight & 12 & 63.2 & 10 & 55.6 & \\
\hline Hypertension Status & & & & & 0.181 \\
\hline Yes & 2 & 10,5 & 5 & 27,8 & \\
\hline No & 17 & 89.5 & 13 & 72.2 & \\
\hline History of Hypertension & & & & & 0.316 \\
\hline Yes & 3 & 15.8 & 1 & 5.6 & \\
\hline No & 16 & 84.2 & 17 & 94.4 & \\
\hline parity & & & & & 0.332 \\
\hline Nullipara & 5 & 26.32 & 9 & 50.00 & \\
\hline Primipara & 11 & 57.89 & 7 & 38.89 & \\
\hline Multipara & 3 & 15.79 & 2 & 11.11 & \\
\hline Family DM History & & & & & 0.181 \\
\hline Yes & 2 & 10.5 & 5 & 27.8 & \\
\hline No & 17 & 89.5 & 13 & 72.2 & \\
\hline History of macrosomia & & & & & 0.580 \\
\hline Yes & 2 & 10.5 & 1 & 5.6 & \\
\hline No & 17 & 89.5 & 17 & 94.4 & \\
\hline
\end{tabular}

Table 2. Mean Changes in HOMA-IR Levels Before and After Intervention

\begin{tabular}{cccccccc}
\hline Variable & Groups & observation & mean \pm SD & MD & \multicolumn{2}{c}{ 95\% CI } & Pvalue \\
\cline { 3 - 4 } & & & & & Lower & Upper & \\
\hline HOMA- & Intervention & Pre & $18.89 \pm 8.49$ & -9.15 & -14.00 & -4.30 & $<0.001$ \\
\cline { 2 - 4 } & & Post & $9.74 \pm 8.62$ & & & & \\
\cline { 2 - 4 } & Control & Pre & $22.89 \pm 12.11$ & 3.42 & -3.95 & 10.80 & 0.341 \\
\cline { 2 - 4 } & & Post & $26.32 \pm 9.31$ & & & & \\
\hline
\end{tabular}

Table 3. Relationship between SaSo Program Intervention on Insulin Resistance (HOMA-IR)

\begin{tabular}{|c|c|c|c|c|c|c|}
\hline \multirow[t]{2}{*}{ Variables } & \multirow[t]{2}{*}{ Groups } & \multirow[t]{2}{*}{ Mean \pm SD } & \multirow[t]{2}{*}{$\Delta$ mean } & \multicolumn{2}{|c|}{ [95\% CI] } & \multirow[t]{2}{*}{$P$-value } \\
\hline & & & & Lower & Upper & \\
\hline HOMA-IR & $\begin{array}{l}\text { - Intervention (SaSo exercise) } \\
\text { - Control (not SaSo } \\
\text { exercise) }\end{array}$ & $\begin{array}{c}-9.15 \pm 10.06 \\
3.42 \pm 14.84\end{array}$ & -12.58 & -20.99 & -4.16 & 0.004 \\
\hline
\end{tabular}

\section{Figures}




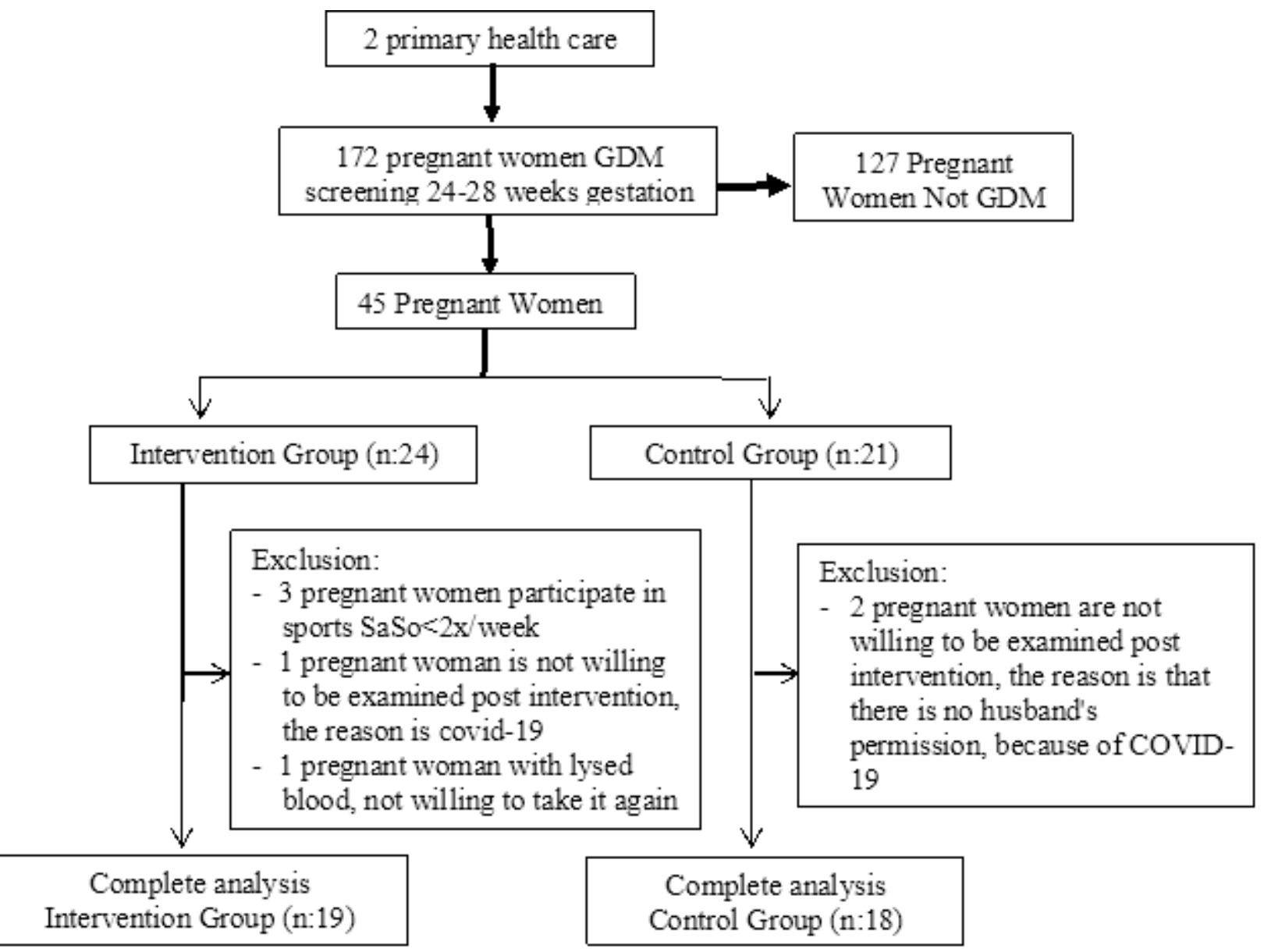

Figure 1. Flowchart of participation in social nervous exercise intervention.

Figure 1

Flowchart of participation in social nervous exercise intervention. 